\title{
ELEMENTS OF ENRICHED AND QUANTUM CATEGORY THEORY
}

\author{
DIMITRI CHIKHLADZE
}

(Received 23 October 2010)

2010 Mathematics subject classification: primary 18D35; secondary 81R50, 18E20.

Keywords and phrases: quantum group, monoidal category, enriched category.

The thesis consists of five research papers. All of them are based on a monoidal category $\mathcal{V}$. The first [1] generalizes the famous embedding theorem for ordinary categories to categories with their homs enriched in $\mathcal{V}$. All the others are dedicated to the study of quantum categories in $\mathcal{V}$. The first paper introduces regular enriched categories and proves an enriched version of the famous embedding theorem by M. Barr, which also generalizes the Freyd-Mitchell embedding theorem for abelian categories.

In the second paper [2] it is shown explicitly how the two known definitions of quantum categories are equivalent. It defines the notion of functor between quantum categories. The outcome is a category qCat. Furthermore, it develops various technical tools for working with quantum categories.

The third paper [5], with Steve Lack and Ross Street, develops a fragment of Hopf algebra theory in the context internal to an autonomous monoidal bicategory in the sense of the formal theory of (co)monads. Specifically, it defines Hopf comonads as monoidal comonads satisfying a certain property corresponding to the property of bialgebra that the fusion map is invertible. This leads to a new (logically equivalent) definition of quantum groupoid.

The fourth paper [3] studies the Takeuchi product $\times_{A}$, first considering an abstract setting of a general monoidal category $\mathcal{V}$, and then covering some special cases. The concept of module for 'many object' generalizations of Hopf structures is discussed. Such modules can also be thought of as quantum analogues of profunctors between small categories.

The fifth paper [4] shows how the Tannaka theory of bialgebroids is related to weak bialgebras, which are the same as bialgebroids with a separable Frobenius base

Thesis submitted to Macquarie University for the degree of Doctor of Philosophy, July 2010. Degree approved, September 2010. Supervisor: Professor Ross Street.

(C) 2011 Australian Mathematical Publishing Association Inc. 0004-9727/2011 \$16.00 
monoid. A Tannaka representation theorem is obtained for a separable Frobenius fiber functor, which is the appropriate kind of fiber functor for the Tannaka theory of weak bialgebras.

\section{Acknowledgements}

Foremost I want to thank my supervisor, Professor Ross Street, whose enduring support and advice during my PhD candidature were incredible. Dr. Steve Lack is a coauthor of one of the papers included in this thesis. I want to thank him. I am grateful to Professor George Janelidze of the University of Cape Town for his attention and hospitality during my visit to his institution. I am grateful to Dr. Brian Day for many concise and insightful mathematical letters I received from him. Support of my associate supervisor, Professor Michel Johnson, is gratefully acknowledged. My appreciation goes to the members and visitors of the Australian Category Seminar. The seminars in category theory held at Macquarie University were a valuable source of inspiration to me. During my PhD I was financially supported by an International Macquarie University Research Scholarship (IMURS) and by the Macquarie University and the Faculty of Science postgraduate research funds.

\section{References}

[1] D. Chikhladze, 'Barr's embedding theorem for enriched categories', J. Pure Appl. Algebra, to appear, arXiv:0903.1173.

[2] D. Chikhladze, 'A category of quantum categories', Theory Appl. Categ. 25 (2011), 1-37.

[3] D. Chikhladze, 'Quantum modules', Preprint, arXiv:1008.1399.

[4] D. Chikhladze, 'Tannaka representation theory with respect to a Frobenius functor', Preprint, arXiv:1008.1400.

[5] D. Chikhladze, R. Street and S. Lack, 'Hopf monoidal comonads', Theory Appl. Categ. 24 (2010), 554-563, arXiv:1002.1122.

DIMITRI CHIKHLADZE, Macquarie University, NSW, Australia e-mail: d.chikhladze@gmail.com 